\title{
Experimental study of the electromagnetic component of drift-wave turbulence
}

\author{
K Rahbarnia*, ${ }^{1}$ E Holzhauer, ${ }^{1} \mathrm{~N}$ Mahdizadeh, ${ }^{2} \mathrm{M}$ Ramisch, ${ }^{1}$ and $\mathrm{U}$ Stroth ${ }^{1}$ \\ ${ }^{1}$ Institut für Plasmaforschung, Universität Stuttgart, 70569 Stuttgart, Germany \\ ${ }^{2} A B B$ Switzerland Ltd, Corporate Research, \\ CH-5405 Baden-Dättwill, Switzerland
}

\begin{abstract}
The magnetic turbulent fluctuation spectrum is investigated in the core of a toroidally confined plasma with parameters similar to those in the fusion edge plasma. The measured spectra show fully developed turbulence over almost 6 decades down to the range of nano-Tesla field amplitudes. The magnetic fluctuations are interpreted as being due to the parallel dynamics of drift-wave turbulence. The predicted strong $\beta$ dependence of the magnetic fluctuations is experimentally confirmed. For the present parameters, anomalous transport due to the magnetic turbulence can be neglected compared to the electrostatic contribution.
\end{abstract}

(Some figures in this article are in colour only in the electronic version.)

*actual address: MPI für Plasmaphysik, EURATOM Association, 17491 Greifswald, Germany E-mail: Kian.Rahbarnia@ipp.mpg.de 


\section{INTRODUCTION}

The contribution of magnetic-field fluctuations to turbulent transport is a long-standing problem in fusion research [1-3]. While electromagnetic turbulence is known to play a decisive role in the high- $\beta$ plasmas of reversed-field pinches $[4,5]$, there is no clear experimental signature yet that it is of importance for the observed level of turbulent transport in tokamak or stellarator plasmas. However, besides the fundamental interest in electromagnetic turbulence it is still a candidate to play a role in electron-heat transport and for the $\beta$ dependence of the transport coefficients.

Magnetic fluctuations can be present in the plasma even in case of predominantly electrostatic turbulence provided the structure of the fluctuations is three dimensional. The coupling between fluctuations in density $\tilde{n}$, electrostatic potential $\tilde{\phi}$ and magnetic field $\tilde{B}$ is caused by a parallel pressure gradient which is a characteristic of drift-wave turbulence. The pressure gradient drives a parallel current resulting in magnetic field fluctuations $\tilde{\mathbf{B}}_{\perp}$ perpendicular to the background field $\mathbf{B}_{\mathbf{0}}$. These fluctuations can in principle lead to a breaking up of the magnetic flux surfaces and thus to enhanced transport [6].

The importance of parallel currents and the concomitant magnetic field component was realised in early work on drift-wave instabilities. The possibility of coupling with Alfvén waves has led to a set of equations describing drift-Alfvén turbulence. This effect was then confirmed in linear plasma experiments $[7,8]$. Recent theoretical work on electromagnetic turbulence can be found e.g. in Refs. [9-12].

This work presents a quantitative experimental study of the electromagnetic turbulent spectrum in the core of a plasma with closed magnetic flux surfaces, at plasma parameters dimensionally similar to those in the edge of fusion plasmas. Previous studies have shown that the characteristics of turbulence in the TJ-K plasma are consistent with drift-wave turbulence by measuring the phase between density and potential fluctuations [13]. The topology of the fluctuations has been shown to be three dimensional [14] with a finite parallel pressure gradient. Hence, parallel current fluctuations are expected in the plasma. Clearly the next step was to use inductive probes to measure the concomitant magnetic fluctuations inside the separatrix and to compare the results with drift-Alfvén-turbulence simulations from the DALF3 code [11], which has been used previously for comparison with TJ-K data (see e.g. [15]). The contribution to turbulent transport is expected to be small in the present 
low- $\beta$ plasma for the range of discharge parameters used in our investigations.

Numerical modeling of collisional drift-wave turbulence has shown that for sufficiently low values of $\beta$ the linear growth rate and the saturation level remain almost unchanged from the purely electrostatic case [16]. Nevertheless weak magnetic fluctuations should be a good test of the predictions from a numerical drift-wave model allowing to study the genesis of these fluctuations.

In fusion plasmas, measurements of the small-scale turbulent magnetic fluctuations are difficult to carry out since probes cannot be inserted into the plasma core. Localised measurements were possible e.g. in the edge of the MST reversed field pinch. It was found that in this configuration, magnetic fluctuations can drive a significant amount of the energy flux [4]. Magnetic probes were also used to measure the magnetic fluctuations on open field lines in the scrape-off layer (SOL) of the small tokamak CASTOR [17]. In this case, currents are driven by flute like instabilities which are electrically connected via the conducting limiter. Other examples for magnetic broadband turbulence measurements with radially movable probes have been reported for the SOL of tokamak experiments, e.g. TEXT [18] and ASDEX [19]. Most of the studies on magnetic turbulence are performed, however, with Mirnov coils located well outside the last closed flux surface (LCFS) of the plasma. As in the Doublet III [20] and COMPASS-D tokamaks [21], this type of diagnostic is preferentially used to study broadband (high m-number) turbulence in the plasma edge or coherent (low m-number) phenomena like Alfvén modes. It appears unlikely that a direct correlation with possible drift-wave turbulence in the plasma core can be thus established. In the TJ-K stellarator, measurements can be performed on closed flux surfaces inside the separatrix, where the turbulence has been shown to be due to collisional drift waves.

In addition to inductive probes, alternative methods have been developed to measure core fluctuations, e.g. cross-polarisation scattering in the Tore Supra tokamak [22]. In section VII the magnetic fluctuation measurements in Tore Supra are briefly discussed. Another method developed recently to measure magnetic fluctuations in the plasma core uses a heavy ion beam probe [23].

The paper is organized as follows: After an introduction into the experiment and diagnostic systems the drift-Alfvén turbulence code DALF3 is briefly discussed. The code constitutes the basis for a comparison of experimental data with numerical simulations. Subsequently the measured and simulated magnetic and electrostatic spectra are presented. 
A qualitative and quantitative analysis of the $\beta$ dependence of magnetic fluctuations followed by a summary and conclusion complete this paper.

\section{EXPERIMENTS AND BACKGROUND PLASMA PARAMETERS}

The experiments have been carried out on the torsatron TJ-K with a major radius of $R_{0}=$ $0.6 \mathrm{~m}$ a minor radius of $a=0.1 \mathrm{~m}$ and a rotational transform of $t \approx 0.3$ [24]. The toroidally confined low-temperature plasma has electron temperatures of $T_{e} \leq 20 \mathrm{eV}$, densities in the range of $n=1-10 \times 10^{17} \mathrm{~m}^{-3}$ and cold ions $\left(T_{i} \leq 1 \mathrm{eV}\right)$. Plasma heating is done with microwaves at $2.45 \mathrm{GHz}$ and $8.2 \mathrm{GHz}$ with heating powers of $1.8 \mathrm{~kW}$ and $0.6 \mathrm{~kW}$, respectively. The two frequencies provide the possibility to work at two values of the background field $B_{0}\left(B_{l}=72 \mathrm{mT}\right.$ and $\left.B_{h}=276 \mathrm{mT}\right)$ to test an important property of magnetic turbulence, namely the $\beta$ dependence of their fluctuation amplitudes. The working gases used here are hydrogen, helium and argon. The relatively low heat load on probes inserted into the plasma allows to average the probe data over many seconds.

The background plasma profiles are measured with scanning Langmuir probes. Fig. 1 depicts radial density and temperature profiles measured for the two values of the magnetic field. The temperature profile is slightly hollow with values of about $8 \mathrm{eV}$ and the density increases from 2 to $6 \times 10^{17} \mathrm{~m}^{-3}$ at the high field. Therefore the core $\beta$ value is reduced by about a factor of 5 when the magnetic field is increased from $B_{l}$ to $B_{h}$.

Because of the extremely low magnetic fluctuation level to be detected, considerable care was taken to optimise the shielding of the probes against electrostatic pickup. The probes consist of loops $(\phi=4 \mathrm{~mm})$ with 20 windings of coated copper wire $(\phi=150 \mu \mathrm{m})$ to measure the poloidal and radial component of the magnetic fluctuations. Each loop has an inductance of $L_{P}=4 \mu \mathrm{H}$ and a resistance of $2 \Omega$. The limiting frequency is $\omega_{0}=R_{T} / L_{P}=2$ $\mathrm{MHz}$ with a terminating impedance of $R_{T}=50 \Omega$. The probe is surrounded by a closed ceramic tube with a diameter of $15 \mathrm{~mm}$ and a length of $l=580 \mathrm{~mm}$. The twisted copper wire is shielded by a slitted grounded metallic foil and connected to an operational amplifier with symmetric input. The amplitude and frequency response of the probe including the amplifier was calibrated in a Helmholtz coil. A double probe with two perpendicularly oriented coils was used (Fig. 2). Hence, radial and poloidal components were measured at the same probe position. At the lower frequency end, the spectra are cut off by a high-pass filter at $5 \mathrm{kHz}$ to 
avoid unwanted contributions from ripples in the power supply feeding the magnetic coils.

The sensitivity of the detection system is defined by the amplifier noise without plasma. Note that the frequency spectra are given as the spectral power density, i.e. the spectral power within a given frequency interval $\Delta f$ which determines the spectral resolution. Unless otherwise noted $\Delta f$ is chosen to be $1 \mathrm{kHz}$. For frequencies above of $20 \mathrm{kHz}$, the amplifier noise lies in the range of $0.1 \mu \mathrm{V}$, which corresponds to a detection limit of $10^{-11}$ Tesla. It is instructive to compare the detection limit of the diagnostic with the thermal fluctuation level which constitutes the lowest possible level in the absence of instabilities [25]. The thermal level of magnetic fluctuations is estimated on the order of $10^{-11} \mathrm{~T}$.

An important point is the possible disturbance of the plasma upon insertion of the probe: Fig. 3 shows radial density (a) and floating potential profiles (b) which have been measured with a Langmuir probe for different radial locations of the magnetic probe inside the plasma. Although the inductive pickup probe was moved up to $20 \mathrm{~cm}$ into the plasma, no significant effect on the profiles can be observed. As shown on the rhs of Fig. 3, the same result holds for the density (3c) and potential fluctuation spectra (3d) measured in the gradient region of the profiles.

\section{TURBULENCE SIMULATIONS}

As in previous work on TJ-K, the experimental results are to be compared with numerical simulations from the DALF3 code. The drift-Alfvén turbulence code DALF3 [26] solves a two-fluid model in 3-dimensional flux-tube geometry. The model includes interchange and drift-wave turbulence, which is coupled to shear-Alfvén waves. The dependent variables are fluctuations in potential $\tilde{\phi}$, electron pressure $\tilde{p}_{e}$, parallel current $\left(\tilde{J}_{\|}\right)$and parallel ion flow $\left(\tilde{u}_{\|}\right)$. The equations read

$$
\begin{array}{r}
\frac{n_{e} M_{i} c^{2}}{B^{2}} \frac{\mathrm{d}}{\mathrm{dt}} \nabla_{\perp}^{2} \tilde{\phi}=\nabla_{\|} \tilde{J}_{\|}-\mathcal{K}\left(\tilde{p}_{e}\right), \\
\frac{n_{e} e}{c} \frac{\partial}{\partial t} \tilde{A}_{\|}+\frac{m_{e}}{e} \frac{\mathrm{d}}{\mathrm{dt}} \tilde{J}_{\|}=\nabla_{\|}\left(p_{e}+\tilde{p}_{e}\right)-n_{e} e \nabla_{\|} \tilde{\phi}-0.51 \frac{m_{e}}{e} \nu_{e} \tilde{J}_{\|}, \\
\frac{\mathrm{d}}{\mathrm{dt}}\left(\tilde{p}_{e}+p_{e}\right)=\frac{T_{e}}{e} \nabla_{\|} \tilde{J}_{\|}-p_{e} \nabla_{\|} \tilde{u}_{\|}-\frac{T_{e}}{e} \mathcal{K}\left(\tilde{p}_{e}\right)+p_{e} \mathcal{K}(\tilde{\phi}), \\
n_{i} M_{i} \frac{\mathrm{d}}{\mathrm{dt}} \tilde{u}_{\|}=-\nabla_{\|}\left(p_{e}+\tilde{p}_{e}\right) .
\end{array}
$$


They represent the conservation of charge, parallel electron and ion momentum and energy, respectively, where

$$
\frac{\mathrm{d}}{\mathrm{dt}}=\frac{\partial}{\partial t}+\frac{c}{B^{2}} \mathbf{B} \times \nabla \phi \cdot \nabla, \quad \mathcal{K} \equiv-\nabla \cdot \frac{c}{B^{2}} \mathbf{B} \times \nabla .
$$

$M_{i}, m_{e}, p_{e}, e$ and $c$ are the ion and electron mass, the background electron pressure, the elementary charge and the velocity of light, and $\nu_{e}$ is the Braginskii electron-collision frequency [27]. The DALF3 code constitutes a first principle simulation using toroidally symmetric flux-tube geometry which here is compared with a real plasma in the three-dimensional toroidal magnetic configuration of the stellarator. The equations are solved on a flux tube modeling a tokamak geometry with closed flux surfaces.

In the normalized form the numerical model is controlled by the dimensionless parameters

$$
\hat{\nu}=0.51 \nu_{e} \frac{L_{\perp}}{c_{s}}, \quad \hat{\beta}=\frac{4 \pi n_{e} T_{e}}{B^{2}}\left(\frac{R_{0}}{t L_{\perp}}\right)^{2}, \quad \hat{\mu}=\frac{m_{e}}{M_{i}}\left(\frac{R_{0}}{t L_{\perp}}\right)^{2}
$$

which are the effective collisionality, the normalized beta and the electron-ion mass ratio, respectively. Here $c_{s}=\sqrt{T_{e} / M_{i}}$ is the sound speed, $L_{\perp}$ the density fall-off length. All spatial variables are normalized to the scaling parameter $\rho_{s}=\sqrt{M_{i} T_{e}} /\left(e B_{0}\right)$.

The vector potential is related to the parallel current through Ampère's law, which reads in the normalized version:

$$
\frac{\tilde{J}_{\|}}{n e c_{s}}=-\rho_{s}^{2} \nabla_{\perp}^{2} \frac{\tilde{A}_{\|}}{B_{0} \rho_{s} \beta} .
$$

Since TJ-K possesses a different magnetic topology, differences between calculated and measured spectra may be expected which will be discussed in section VI. Furthermore DALF3 calculates a local solution which means that the background parameters and thus the dimensionless parameters in Eq. 6 must remain constant over the numerical grid. As an example, for He with $B_{0}=72 \mathrm{mT}$ the radial extent of the numerical grid is $10 \mathrm{~cm}$. Over this distance variations in $n_{e}=n$ cannot be neglected (cf. Fig. 1). Thus the input parameters for the calculations represent an approximation to the experimental plasma profile.

In order to allow a quantitative analysis, the probe response has been simulated in the code by calculating the induced voltage from $U_{\text {ind }}=-\int\left(\boldsymbol{\nabla} \times\left(\mathrm{d} \mathbf{A}_{\|} / \mathrm{d} t\right)\right) \mathrm{d} \mathbf{S}$, where the integral has to be taken over the probe cross-section translated into the dimensionless parameter of the code. In principle, a finite area of integration acts as a low-pass filter on the wavenumber spectrum of the fluctuations. Therefor the influence of the numerical probe size on the spectra has been checked. Increasing the size from the smallest possible dimension of 1 grid 
point to the physical value of 3 corresponding to the probe diameter results in a change of less than 5\%. Thus for the dimensions of our magnetic probe this effect can be neglected.

\section{THE $\beta$ DEPENDENCE OF MAGNETIC FLUCTUATION AMPLITUDES}

In addition to the numerical code, one can obtain simple scaling laws from linearized equations for parallel current and concomitant magnetic field fluctuation amplitudes. The prominent relation is the $\beta$ dependence of the ratio of magnetic to potential fluctuation amplitudes. The theoretical basis of the $\beta$ scaling is Eq. 1 in the linearized form without the curvature term:

$$
\frac{\rho_{s}}{c_{s}} \frac{\partial}{\partial t} \rho_{s}^{2} \nabla_{\perp}^{2}\left(\frac{e \tilde{\phi}}{T_{e}}\right)=\rho_{s} \nabla_{\|} \frac{\tilde{J}_{\|}}{n e c_{s}} .
$$

Inserting Eq. 7 for the parallel current, the equation can be rewritten in Fourier space as

$$
\frac{\tilde{B}}{B_{0}}=-\frac{\omega}{k_{\|} c_{s}}\left(k_{\perp} \rho_{s}\right) \beta \frac{e \tilde{\phi}}{T_{e}} \sim \beta \frac{e \tilde{\phi}}{T_{e}} \approx \beta \frac{\tilde{n}}{n},
$$

where $\tilde{B}=k_{\perp} \tilde{A}_{\|}$and the Boltzmann relation $(e \tilde{\phi}) / T_{e} \approx \tilde{n} / n$ are used. The parallel wavenumber $k_{\|}$is prescribed by the torus geometry [14]. Here the maximum power in the fluctuations is assumed to reside at $k_{\perp} \rho_{s}=$ const.. Furthermore, the characteristic frequency of drift waves is given by $c_{s} / L_{\perp}$. Since the temperature $T_{e}$ and $c_{s}$ are taken constant while $\beta$ is varied, the expression $\left(\omega / k_{\|} c_{s}\right)\left(k_{\perp} \rho_{s}\right)$ in Eq. 9 is also constant. Hence, the ratio of the fluctuation power in magnetic and potential $R=\left(\tilde{B} / B_{0}\right)^{2} /\left(e \tilde{\phi} / T_{e}\right)^{2}$ or density fluctuations $R=\left(\tilde{B} / B_{0}\right)^{2} /\left(\tilde{n} / n_{0}\right)^{2}$ is expected to scale as $R_{\phi, B} \approx R_{n, B} \sim \beta^{2}$.

In the experiment, the $\beta$ scaling was investigated making use of the two available values of the magnetic field. The coefficients for e.g. the density at low and high magnetic field $R_{n, B_{l}}$ and $R_{n, B_{h}}$, respectively, can be combined to eliminate the proportionality constant. To this end the quantity $Q_{n} \equiv\left(R_{n, B_{l}} / R_{n, B_{h}}\right)^{1 / 2}=\left(\beta_{l} / \beta_{h}\right)$ is defined, which corresponds to a measurement of the $\beta$ scaling of the fluctuation amplitudes. All measurements are performed in the gradient region at $r=R-R_{0}=0.1 \mathrm{~m}$, where density and temperature are taken to be constant for the main fields of $B_{l}$ and $B_{h}$. One therefore can expect a change in the ratio of the amplitudes by a factor of $Q_{n}^{\text {theo }}=Q_{\phi}^{\text {theo }} \approx\left(B_{h} / B_{l}\right)^{2} \approx 15$. 


\section{V. $\beta$ SCALING IN DALF3 SIMULATIONS}

As an example, for the parameters at helium discharges the value of $\hat{\beta}$ in the experiment decreases from 0.02 to the extremely small value 0.003 . For $\hat{\beta}=0.003$ the DALF3 code can no longer temporally resolve the appendant shear-Alfvén waves ([26], Eq. 23). To circumvent this problem in the simulations of the $\beta$ scaling the values of $\hat{\beta}$ were increased to $\hat{\beta}=0.05$ and $\hat{\beta}=0.5$ while keeping the ratio unchanged.

Fig. 4 shows a comparison of electrostatic and magnetic fluctuation spectra from DALF3 simulations for these two values of $\hat{\beta}$. The gray line repeating the density spectrum serves as a reference. The arrows indicate the factors between the electrostatic and magnetic fluctuations which are $1 \times 10^{8}$ and $1 \times 10^{6}$ for the low and high $\hat{\beta}$ case, respectively. In section IV is shown that the ratio of the turbulent electrostatic and magnetic fluctuation power spectra is expected to scale with $\beta^{-2}$ (see Eq. 9). For the simulations, the values for $\beta$ are $\beta_{h}=1.4 \times 10^{-4}$ and $\beta_{l}=1.4 \times 10^{-3}$ for the high $(\hat{\beta}=0.05)$ and low magnetic field $(\hat{\beta}=0.5)$, respectively (Eq. 6). This leads to the scaling factors of $\beta_{h}^{-2} \approx 5 \times 10^{7}$ and $\beta_{l}^{-2} \approx 5 \times 10^{5}$. The comparison with the estimates from the simulations of the factors shown in Fig. 4 of $1 \times 10^{8}$ and $1 \times 10^{6}$ is satisfactory taking into account the many orders of magnitude spanned. The measure for the $\beta$ scaling $Q_{n}=\left(R_{n, B_{l}} / R_{n, B_{h}}\right)^{1 / 2}$ as calculated from the spectra gives the expected ratio of 10 for these DALF3 simulations.

These results show that the scaling of magnetic turbulence with $\hat{\beta}$ is largely independent of the absolute value of $\hat{\beta}$ as long as one is in the electrostatic regime for the drift-wave turbulence.

\section{MEASURED MAGNETIC FLUCTUATION SPECTRA}

The inductive pickup probe has been used to measure power spectra of the magnetic fluctuations in discharges with different working gases (Ar, He, H) and magnetic field strengths. Fig. 5 depicts examples of frequency spectra measured in the density-gradient region of hydrogen, helium and argon discharges. The spectra exhibit a turbulent cascade over up to 6 decades. The lowest detected magnetic fluctuation level at $250 \mathrm{kHz}$ corresponds to amplitudes in the range of $10^{-9} \mathrm{~T}$. In the argon case, quasi coherent modes are visible in the spectrum, while hydrogen and helium spectra are smooth with spectral indices in the 
wavenumber spectra which lie between -4 and -5 . With a $2 \mathrm{D}$ Langmuir probe array the electrostatic turbulence was found close to isotropic in the poloidal plane in agreement with results from the DALF3 code. In the following, only the results from hydrogen $\left(\rho_{s}^{l}=4 \mathrm{~mm}\right.$, $\left.\rho_{s}^{h}=1 \mathrm{~mm}\right)$ and helium $\left(\rho_{s}^{l}=6 \mathrm{~mm}, \rho_{s}^{h}=1.5 \mathrm{~mm}\right)$ will be used (indices $l, h$ indicate low and high magnetic field, respectively). For argon, the value of $\rho_{s}^{l}=20 \mathrm{~mm}$ is not sufficiently small compared with the gradient length $L_{\perp}=96 \mathrm{~mm}$.

For discharges in helium and hydrogen, Fig. 6 shows the spectra from a magnetic probe (poloidal component) in comparison with spectra of the electrostatic fluctuations as measured with Langmuir probes on an equivalent flux surface. Since the turbulence is of the drift-wave type, the normalized density and potential fluctuations have similar amplitudes in agreement with the Boltzmann relation. The relative decrease in potential fluctuation power with respect to density fluctuations at higher frequencies can be attributed to the cable capacitance. Together with the load impedance this capacitance acts like a low-pass filter. The magnetic fluctuation spectra have a shape similar to the density spectra but the normalized power is considerably lower. This similarity points to the same source as being responsible for both the electrostatic and magnetic type of fluctuations. This is highlighted by the gray line superimposed on the magnetic turbulence spectrum which corresponds to the density spectrum divided by $5 \times 10^{8}$. The upper part of Fig. 6 shows the comparison of the power spectra for $B_{0}=72 \mathrm{mT}$. The bottom part shows the comparison of the power spectra for $B_{0}=276 \mathrm{mT}$ where now the gray line corresponds to the density spectrum divided by $1 \times 10^{11}$. As shown in section IV, the ratio of the turbulent electrostatic and magnetic fluctuation power spectra is expected to scale with $\beta^{-2}$. In case of low $B_{0}$ (Fig. 6 top), the corresponding values are $\beta=5.4 \times 10^{-5}$ for helium and $\beta=7.7 \times 10^{-5}$ for hydrogen. So scaling factors of $3.5 \times 10^{8}$ for helium and $1.7 \times 10^{8}$ for hydrogen follow. In case of high $B_{0}$ (Fig. 6 bottom), the corresponding values are $\beta=0.85 \times 10^{-5}(\mathrm{He})$ and $\beta=0.58 \times 10^{-5}$ $(\mathrm{H})$. Now the scaling factors are $1.4 \times 10^{10}(\mathrm{He})$ and $2.9 \times 10^{10}(\mathrm{H})$. These values compare well with the estimates of the factors given by the ratios in Fig. 6 top and bottom which are $5 \times 10^{8}$ and $1 \times 10^{11}$, respectively. With respect to the many orders of magnitude spanned the agreement between prediction and the experiment is quite good.

The results of radial profile measurements of the fluctuation amplitudes for helium and hydrogen discharges $\left(B_{0}=72 \mathrm{mT}\right)$ are given in Fig. 7 where the normalized fluctuations $(\tilde{n} / n)(\mathrm{r}),\left((e \tilde{\phi}) / T_{e}\right)(\mathrm{r})$ and $\left(\tilde{B} / B_{0}\right)(\mathrm{r})$ are plotted as a function of the radial position $r=$ 
$R-R_{0}$ inside the plasma. As in Fig. 6, similar normalized amplitudes of density and potential fluctuations are found. These amplitudes are largest in the region where the strongest density gradient is located which is on the low-field side of the plasma. On the high-field side, a second smaller maximum is visible which is less pronounced. This result is consistent with observations from a poloidal array with Langmuir probes which shows that the turbulent fluctuations are concentrated on the low-field side. A clear peaking in the gradient region is also visible in the small level magnetic fluctuations. Fig. 7 also illustrates that the radial and poloidal component of the magnetic fluctuations have comparable amplitudes. Like the electrostatic components of the turbulence, the magnetic fluctuations are found to be close to isotropic in the poloidal plane. This confirms the results obtained with the 2D Langmuir probe array for density fluctuations.

Outside the separatrix, the magnetic fluctuation level drops off steeply in contrast to the electrostatic quantities. The low level of magnetic turbulence measured with the movable probe near the separatrix is confirmed by measurements with an array of Mirnov coils mounted on the inner wall. The conclusion is that there is no source for strong magnetic activity in the SOL. This seems to be in agreement with measurements in tokamaks which have shown that the normalized electrostatic fluctuations still increase in the SOL, whereas magnetic turbulence decreases [18]. Note that $\tilde{n}(\mathrm{r})$ and $\tilde{\phi}(\mathrm{r})$ are normalized to plasma background parameters which vary with radius whereas $\tilde{B}$ is normalized to a constant value of $B_{0}$.

Fig. 8 compares experimental and simulated power spectra of the electrostatic and magnetic fluctuations for a helium and a hydrogen discharge. The simulated spectra exhibit marked peaks which are most pronounced in the electrostatic potential. Note that the calculated plasma-potential fluctuations $\tilde{\phi}_{p l}$ are in principle comparable with the measured floating-potential fluctuations $\tilde{\phi}_{f l}$, because in TJ-K $\tilde{\phi}_{p l} \approx \tilde{\phi}_{f l}=\tilde{\phi}[29]$. The well defined resonant frequency, which only occurs in the calculated hydrogen spectra, points to a geodesic acoustic mode (GAM) [30, 31]. DALF3 uses a toroidally symmetric flux tube geometry while the flux surfaces in TJ-K show a complex three-dimensional shape. At present it is not known, whether in such a configuration a GAM mode can exist.

For both gases, the shape of the broadband component in the magnetic and electrostatic spectra is similar. The gray vertical arrows indicate that power in the magnetic component is by about 8 orders of magnitude smaller than in the electrostatic component. It is noteworthy 
that this result holds for both the numerical code and the experiment. The results are given for the local background parameters in the gradient region as shown in Fig. 1 ( $B_{0}=$ $72 \mathrm{mT}$ ). The power of the magnetic turbulence lies mainly at frequencies between 8 and $12 \mathrm{kHz}$. For the discharge in helium, the difference in spectral power at $10 \mathrm{kHz}$ between experiment and simulation is one order of magnitude. Again this may be, at least in part, due to the difference between the flux tube geometry in the numerical code and the 3dimensional stellarator geometry of the experiment. But taking into account the many orders of magnitude spanned the agreement with DALF3 and the experiment is very reasonable.

Next the experimental $\beta$ scaling of the magnetic fluctuations is studied. Fig. 9 depicts normalized experimental frequency power spectra of electrostatic and magnetic fluctuations for helium and hydrogen discharges. The dependence of the spectral power on the toroidal magnetic field $B_{0}$ can clearly be seen. As expected from Eq. 9, the strongest effect observed occurs in the magnetic fluctuations. For both types of gas, their power decreases strongly when the magnetic field is increased. The total power in the corresponding spectra has been calculated. In Tab. I the values for $Q_{n, B}$ and $Q_{\phi, B}$ for the $\beta$ scaling are given. In comparison with the theoretically expected value of $\left(B_{h} / B_{l}\right)^{2} \approx 15$ it turns out that the scaling of the magnetic fluctuation amplitudes found in the experiment is higher by a factor of 1 to 5 for helium and 5 to 22 for hydrogen. At present the higher values for hydrogen are not sufficiently understood. Keeping in mind that these factors are based on the ratio of measured spectra in Fig. 8 which differ by more than 8 orders of magnitude in power, the agreement is still acceptable.

While changing $\beta$ with the variation of the magnetic field the effective collisionality $\hat{\nu}=0.51 \nu_{e} L_{\perp} / c_{s} \sim n_{e} / T_{e}^{3 / 2}$ (see Eq. 6) changes by up to a factor of 3 (see helium discharge profiles in Fig. 1). This is much less than the variation of $\hat{\nu}$ with the plasma radius $r=R-R_{0}$. It varies from $\hat{\nu} \approx 6$ in the plasma centre to $\hat{\nu} \approx 0.4$ at the edge by a factor of 15 . From the measurements of the radial profile of $\tilde{B} / B_{0}$ (Fig. 7 ) a rather small radial change with a factor of approximately 2 is obtained. Hence the dependence of $\tilde{B} / B_{0}$ on $\hat{\nu}$ is much weaker than the dependence of $\tilde{B} / B_{0}$ on the magnetic field. When the magnetic field is increased by a factor of 3.8 from $B_{l}$ to $B_{h}$, the measured normalized magnetic fluctuations decrease by a factor of about 100 (see Fig. 9, He). In addition in the DALF3 simulations $\hat{\nu}$ variations have only a weak influence on the calculated fluctuation amplitudes. This is studied in detail in [15]. 


\section{ESTIMATE OF RESULTING ANOMALOUS TRANSPORT FROM MAG- NETIC FLUCTUATIONS}

The total turbulent particle transport is given by:

$$
\Gamma_{\text {turb }}=-\left(D_{e s}+D_{m a g}\right) \nabla n
$$

The electrostatic diffusion coefficient $D_{e s}=\left(k_{\theta} / B_{0}\right)^{2}|\tilde{\phi}|^{2} \tau_{\text {corr }}$ can be estimated from the characteristic poloidal wavenumber $k_{\theta}=2 \pi / L_{\text {corr }}$ with the correlation length $L_{\text {corr }}$ and a characteristic correlation time $\tau_{\text {corr }}$. The value has been investigated for TJ-K plasmas to be in the range of $D_{e s}=2-20 \mathrm{~m}^{2} / \mathrm{s}$ [28]. An estimate of the magnetic diffusion coefficient can be obtained from $D_{\text {mag }}=R_{0}^{2} /\left(t^{2} \tau\right)\left(\tilde{B}_{r} / B_{0}\right)^{2}$ [1], where $\tau$ is the electron-collision time and $\tilde{B}_{r}$ the radial component of the magnetic fluctuations. From the measured spectra (e.g. in Fig. 5, He) it follows $D_{m a g}=10^{-6}-10^{-5} \mathrm{~m}^{2} / \mathrm{s}$; a value 6 magnitudes smaller than the electrostatic one. As expected for such a low- $\beta$ plasma, the measured fluctuation amplitudes lead to a negligible contribution of the magnetic component to the total turbulent transport.

In plasma discharges with higher $\beta$ like for example in the Tore Supra tokamak [22] magnetic fluctuation amplitudes of typically $\tilde{B} / B_{0} \approx 4 \times 10^{-5}$ have been measured. Compared to typical values in TJ-K $\tilde{B} / B_{0} \approx 1 \times 10^{-6}$ (see Fig. 7 ) the ratio of the normalized magnetic fluctuations is 40 . The authors of [22] found that experimentally observed results in the Tore Supra Tokamak indicate that small scale magnetic fluctuations are a serious candidate for explaining the anomalous heat transport of electrons in the gradient region.

\section{SUMMARY AND CONCLUSION}

The turbulent magnetic spectrum associated with drift-wave turbulence in TJ-K was measured using a highly sensitive pickup probe. The power spectra were detected over a range of more then 6 decades with the lowest value in the nano-Tesla range. The amplitudes of $\tilde{B} / B_{0}$ are about $4-5$ decades lower than those of the normalized electrostatic fluctuations $(e \tilde{\phi}) / T_{e}$ and $\tilde{n} / n$. Nevertheless, the shape of the magnetic spectra agrees well with those of density and potential fluctuations. This confirms the common root of these fluctuations, namely drift-wave turbulence. As an important topic the $\beta$ dependence of the magnetic fluctuation amplitudes has been investigated. The physical processes underlying the scaling 
have been elucidated using a linearized form of the equations defining the parallel current and thereby the fluctuating magnetic field. The measured ratio in power levels between density and magnetic turbulence spans 8-9 orders of magnitude. In view of this large dynamic range, the observed $\beta$ scaling lies sufficiently close to the predicted values.

The experimental data have been compared to numerical simulations with the DALF3 code. Good agreement has been found with the experimental scaling of magnetic and electrostatic turbulence with $\beta$. In summary, the magnetic turbulence in TJ-K has been identified as being due to dominantly electrostatic drift-wave turbulence. The extremely small contribution of the magnetic power to the total turbulent transport underlines the electrostatic character of the turbulence in TJ-K.

\section{ACKNOWLEDGMENT}

Financial support by Max-Planck-Institut für Plasmaphysik EURATOM Ass., Garching and Greifwald is gratefully acknowledged. The authors are also grateful to B. Scott and A. Kendl who made the DALF3 code available and gave helpful advice.

\section{REFERENCES}

[1] Rechester A B and Rosenbluth M N 1978 Phys. Rev. Lett. 40(1) 38-41

[2] Callen J D and Jahns G L 1976 Phys. Rev. Lett. 38491

[3] Rebut P H and Brusati M 1986 Plasma Phys. Control. Fusion 28113

[4] Fiksel G, Prager S C, Pribyl P, Taylor, R J and Tynan G R 1995 Phys. Rev. Lett. 75 3866

[5] Martin P, Marrelli L, Spizzo G et al. 2003 Nucl. Fusion 431855

[6] Callen J D 1977 Phys. Rev. Lett. 391540

[7] Tang J T, Luhmann N C, Nishida Y and Ishii K 1975 Phys. Rev. Lett. 3470

[8] Nishida Y and Ishii K 1974 Phys. Rev. Lett. 33352

[9] Yagi M, Itho S I, Itho K and Fukuyama A 1997 Plasma Phys. Control. Fusion 391887

[10] Hinton F L, Rosenbluth M N and Waltz R E 2003 Phys. Plasmas 10168

[11] Scott B D 2003 Plasma Phys. Control. Fusion 45 A385

[12] Naulin V 2003 Phys. Plasmas 104016 
[13] Stroth U, Greiner F, Lechte C, Mahdizadeh N, Rahbarnia K and Ramisch M 2004 Phys. Plasmas 112558

[14] Mahdizadeh N, Greiner F, Happel T, Kendl A, Ramisch M, Scott B D and Stroth U 2007 Plasma Phys. Control. Fusion 49(7) 1005-1017

[15] Niedner S, Scott B D and Stroth U 2002 Plasma Phys. Control. Fusion 44397

[16] Camargo S J, Scott B D and Biskamp D 1996 Phys. Plasmas 33912

[17] Stökel J et al. 1999 Plasma Phys. Control. Fusion 41 A577

[18] Connor J W 1993 Plasma Phys. Control. Fusion 35 B293-B305

[19] Giannone L 1988 Proc. 15th European Conference on Controlled Fusion and Plasma Heating Dubrovnik III 1143

[20] Ohyabu N, Jahns G L, Stambaugh R D and Strait E J 1987 Phys. Rev. Lett. 58120

[21] Han W E, Thyagaraja A, Fielding S J and Valovic M 2000 Plasma Phys. Control. Fusion 42181

[22] Colas L, Zou X L, Paume M, Chareau J M, Guiziou L, Hoang G T, Michelot Y and Gresillon D 1998 Nucl. Fusion 38903

[23] Fujisawa A, Shimizu A, Nakano H and Ohshima S 2007 Plasma Phys. Control. Fusion 49845

[24] Krause N, Lechte C, Stöber J, Ascasibar E, Alonso J, Niedner S and Stroth U 2002 Rev. Sci. Inst. 733474

[25] Krommes J A 1993 Phys. Fluids B 5(4) 1066

[26] Scott B 1997 Plasma Phys. Control. Fusion 391635

[27] Braginskii S I Review of Modern Physics (Consultants Bureau, New York, 1956) vol. 1 chap. Transport processes in plasmas

[28] Ramisch M, Mahdizadeh N, Greiner F, Lechte C, Rahbarnia K and Stroth U 2005 Phys. Plasmas 12032504

[29] Mahdizadeh N, Greiner F, Ramisch M, Guttenfelder W, Lechte C, Rahbarnia K and Stroth U 2005 Plasma Phys. Control. Fusion 47(4) 569-579

[30] Kendl A and Scott B D 2005 Phys. Plasmas 12052515

[31] Ramisch M, Niedner S, Scott B and Stroth U 2003 New J. Physics 512.1 


\begin{tabular}{c|cc}
\hline \hline He $(\mathrm{H})$ & $Q_{n, B}$ & $Q_{\phi, B}$ \\
\hline poloidal & $77(329)$ & $43(101)$ \\
radial & $32(217)$ & $19(67)$ \\
\hline \hline
\end{tabular}

TABLE I: Results of the scaling of $Q_{n, B}$ and $Q_{\phi, B}$ for the radial and poloidal component of the magnetic fluctuations with helium and hydrogen (in brackets) as the working gases. These numbers has to be compared with the value $Q_{n}^{\text {theo }}=Q_{\phi}^{\text {theo }} \approx 15$. 


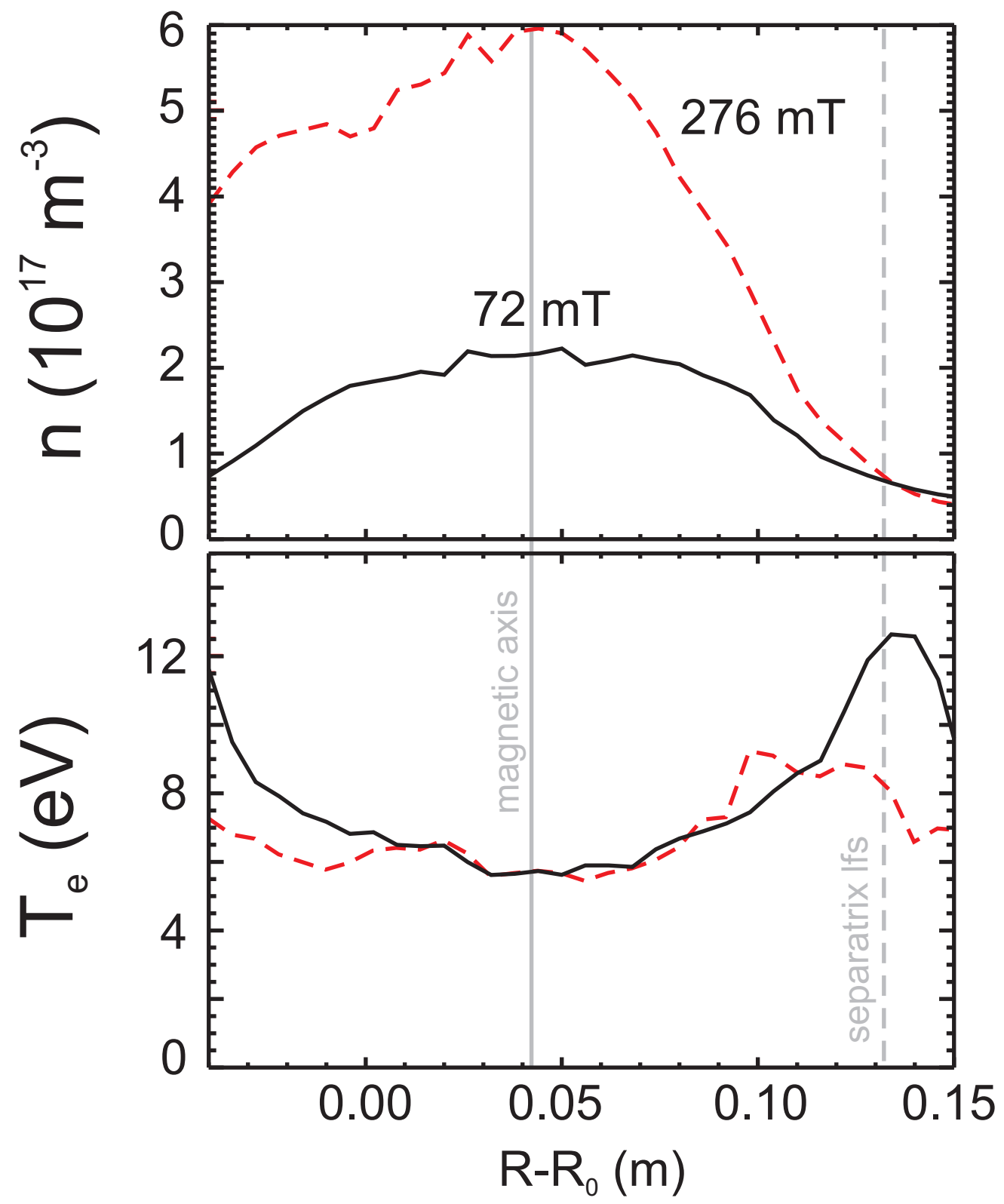

FIG. 1: Background radial density and electron-temperature profiles at two values of the magnetic field $\left(B_{l}=72 \mathrm{mT}\right.$ and $B_{h}=276 \mathrm{mT}$ ) for helium discharges as measured with swept Langmuir probes. The radial position of magnetic axis at $R-R_{0}=4 \mathrm{~cm}$ and separatrix $R-R_{0}=13 \mathrm{~cm}$ are indicated by vertical gray lines. 


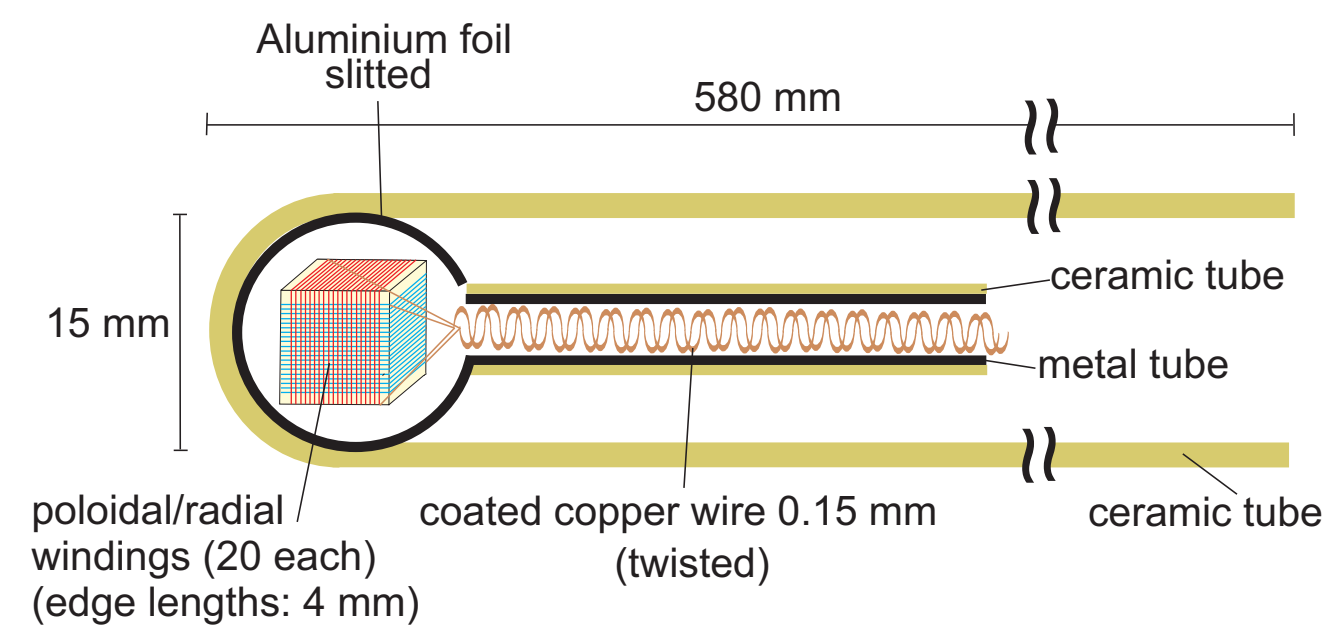

FIG. 2: Magnetic field probe used in the experiment. It consists of two perpendicularly oriented coils. Radial and poloidal fluctuations are measured at the same probe position.
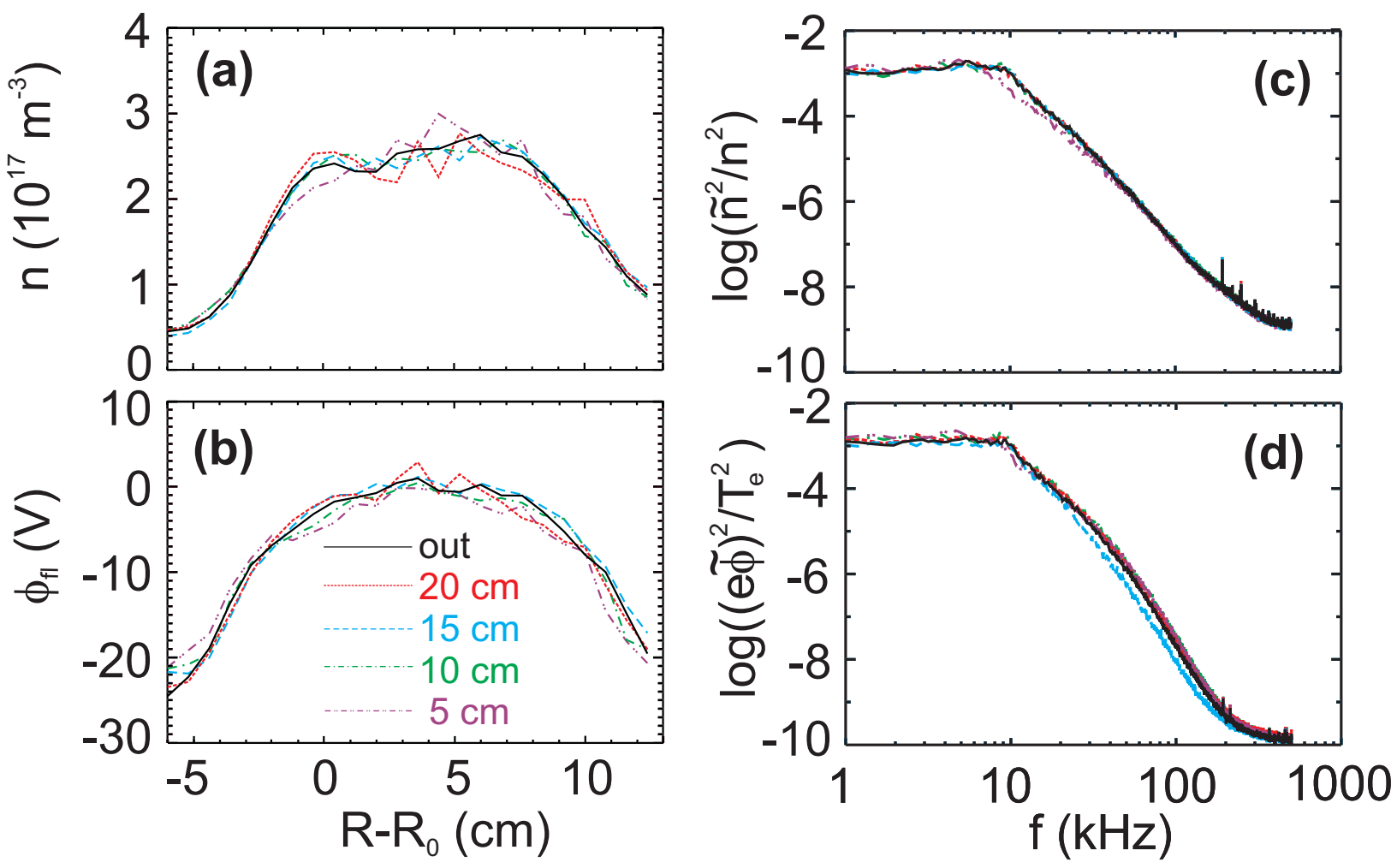

FIG. 3: Radial profiles of density (a), floating potential (b), and the corresponding power spectra (c) and (d) for different positions of the magnetic probe inside the plasma (different linestyles; the solid line (out) means magnetic probe outside the plasma). The profiles are measured with a movable Langmuir probe in a helium discharge; spectra are measured in the density gradient region. 


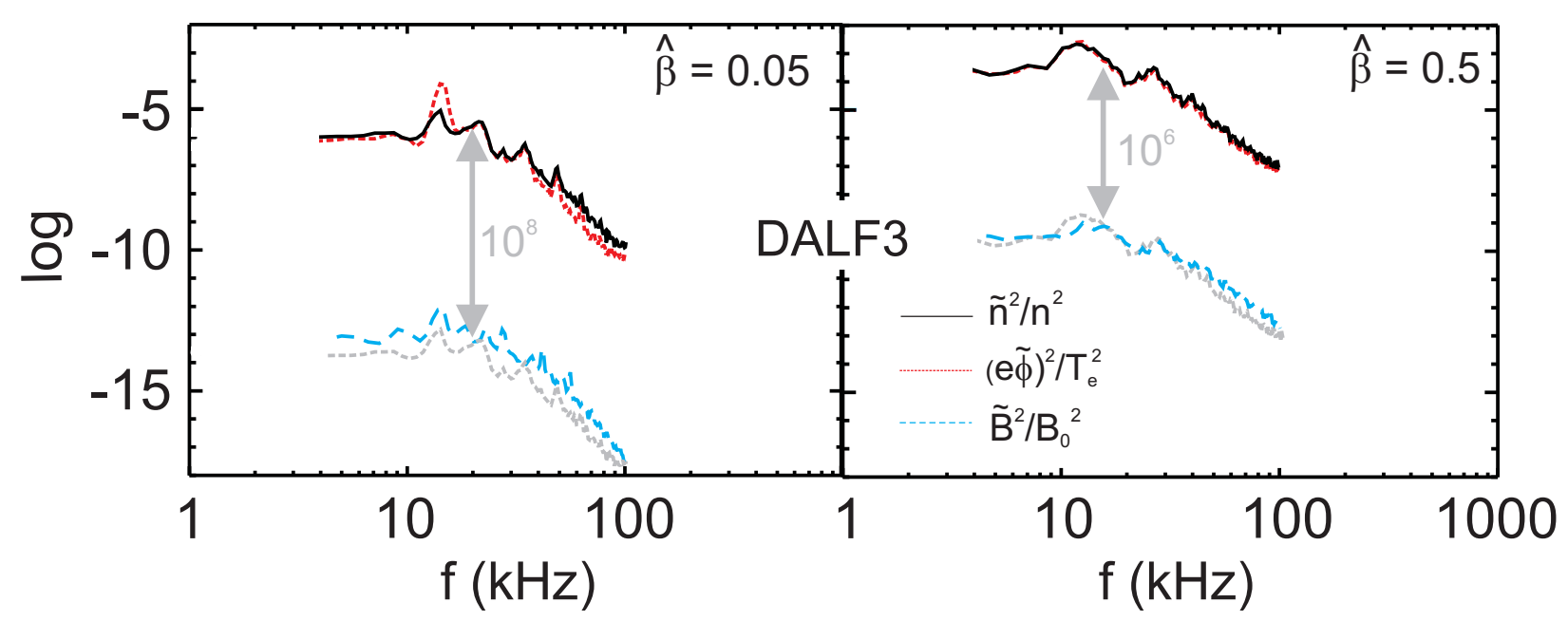

FIG. 4: Comparison of electrostatic and magnetic fluctuation spectra from DALf3 for $\hat{\beta}=0.05$ and $\hat{\beta}=0.5$ (helium simulation). The gray line repeats the density spectrum and serves as a reference. The arrows indicate the factor between the electrostatic and magnetic fluctuations.

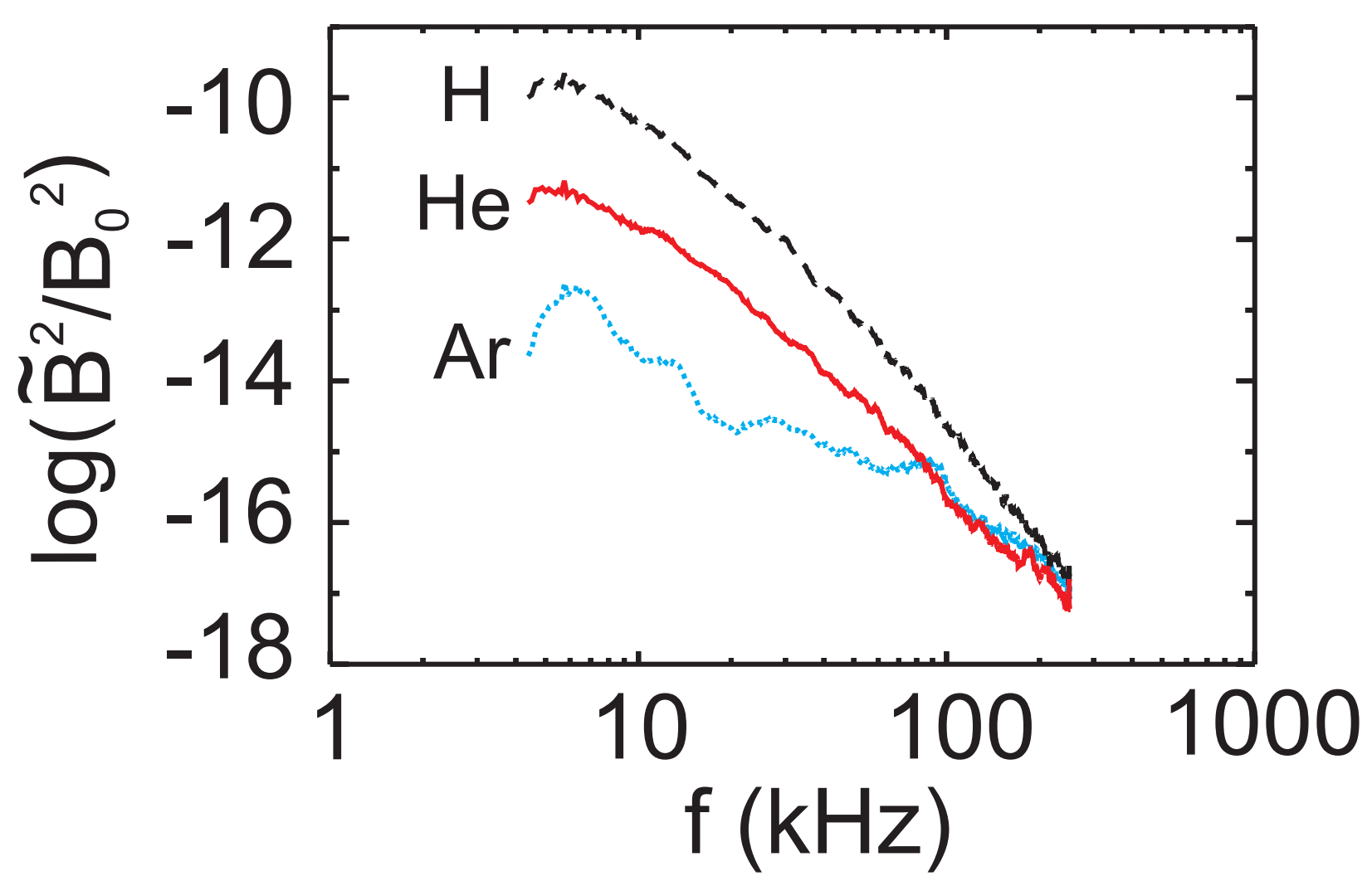

FIG. 5: Magnetic fluctuation spectra measured in the density gradient for the working gases hydrogen, helium and argon at $B_{0}=72 \mathrm{mT}$. 


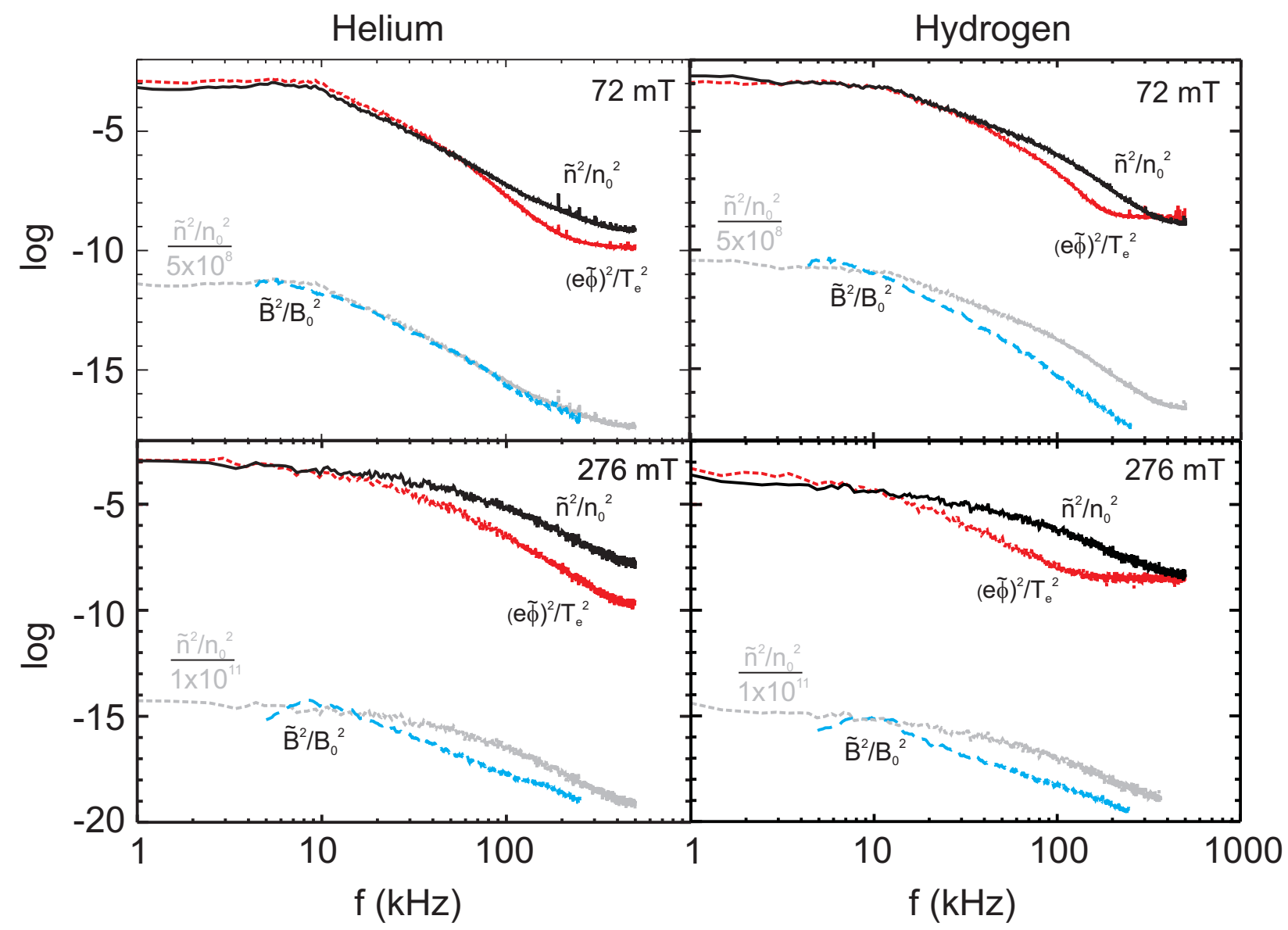

FIG. 6: Comparison of electrostatic and magnetic fluctuation spectra measured in the density gradient region of a helium (left) and hydrogen (right) discharge for $72 \mathrm{mT}$ (top) and $276 \mathrm{mT}$ (bottom) magnetic field. The gray line superimposed on the magnetic fluctuation spectra serves as a reference; it repeats the density spectrum (top) multiplied by $5 \times 10^{8}$ and (bottom) multiplied by $1 \times 10^{11}$. 


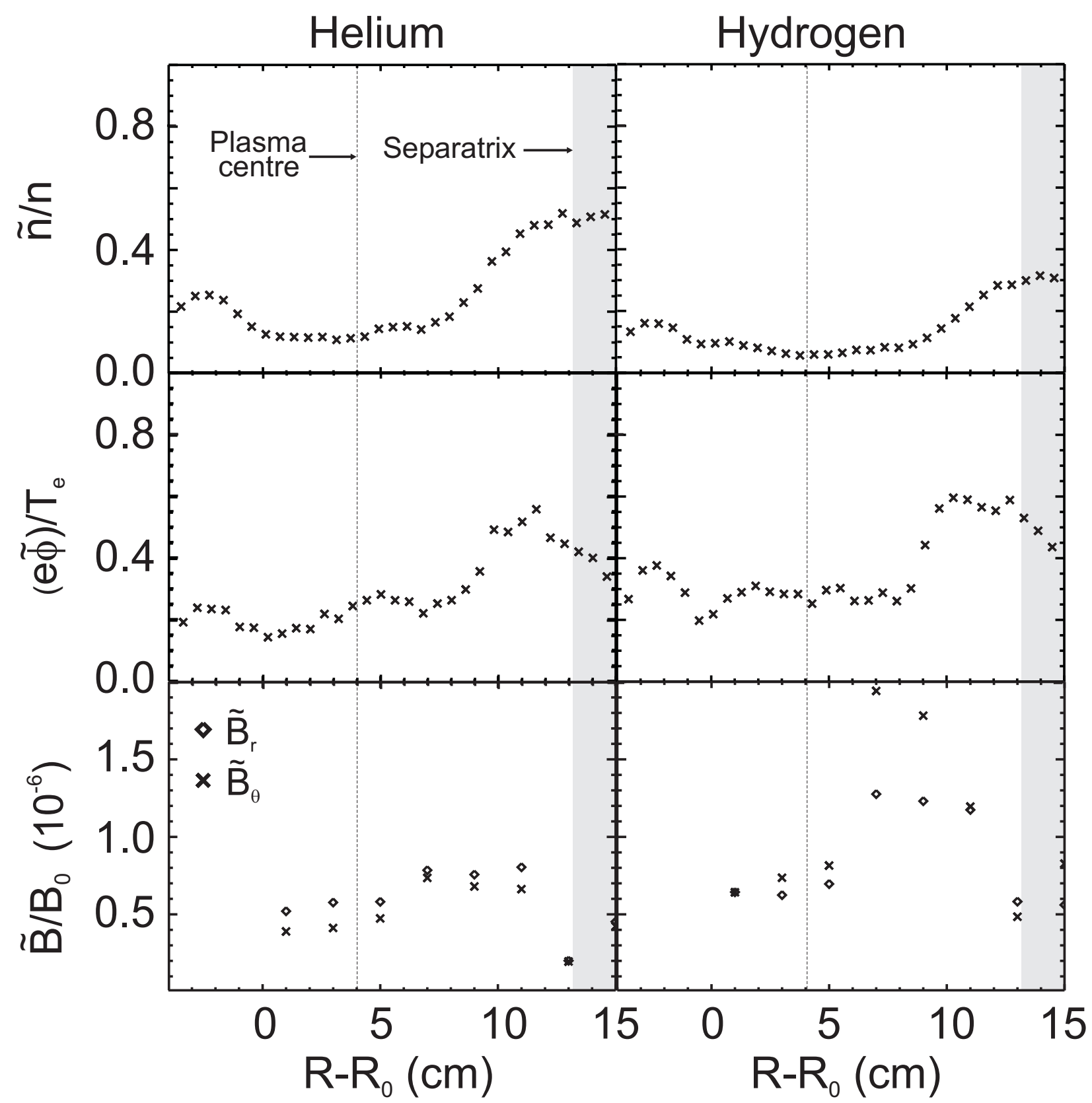

FIG. 7: Radial profiles of electrostatic and magnetic fluctuation amplitudes in a helium (left) and a hydrogen (right) discharge. For the magnetic fluctuations, both radial (diamonds) and poloidal components (crosses) are plotted. The radial position for the magnetic axis is $R-R_{0}=4 \mathrm{~cm}$ and $R-R_{0}=13 \mathrm{~cm}$ for the separatrix. The SOL with open magnetic field lines is shaded in gray. The data have been taken at $B_{0}=72 \mathrm{mT}$. 


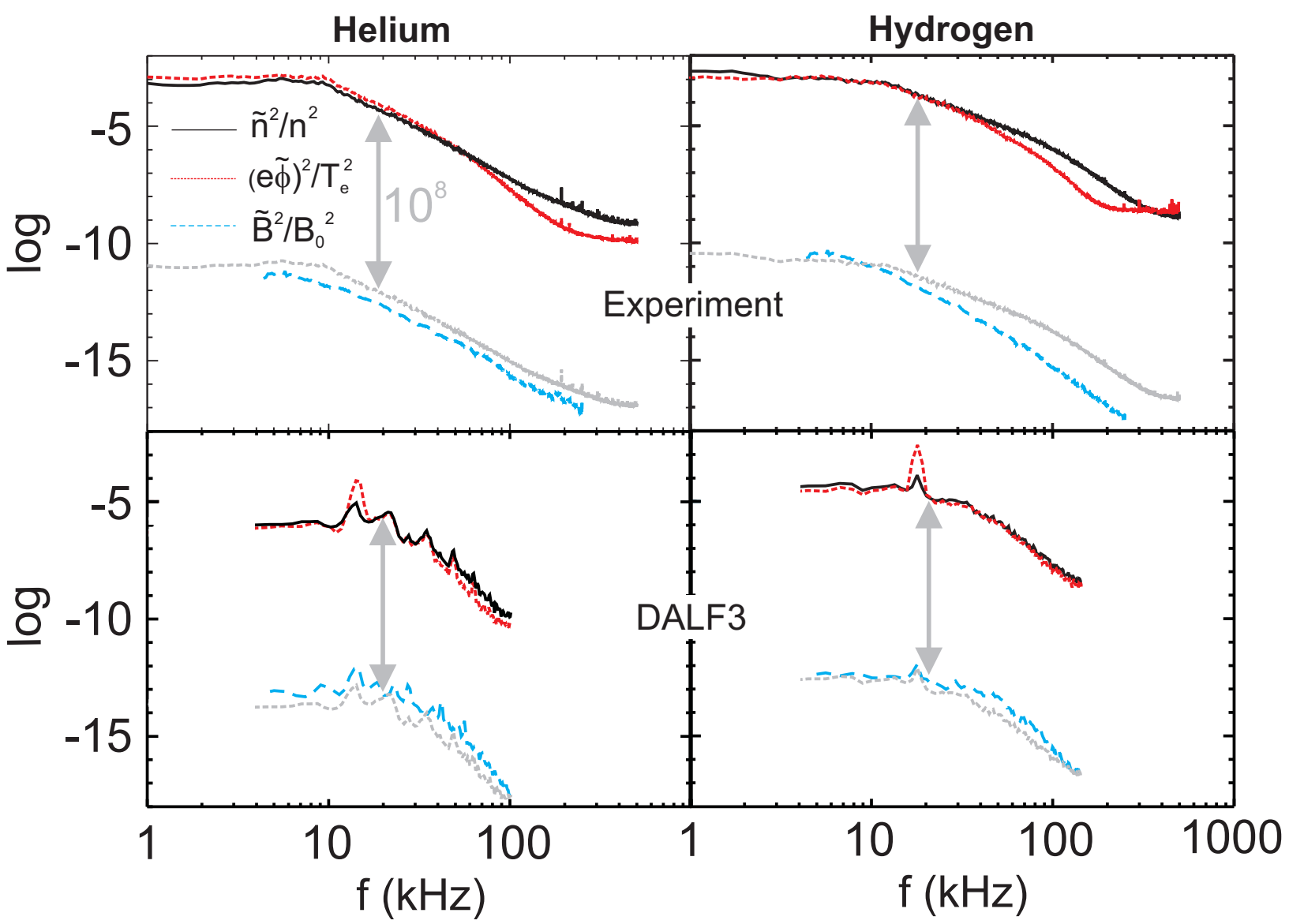

FIG. 8: Comparison of the experimental (top) with simulated (bottom) electrostatic and magnetic fluctuation power spectra (poloidal component) for helium (left) and hydrogen (right) discharges. The calculations were performed with the DALF3 code. A factor $10^{8}$ is indicated by the vertical gray arrows and as reference the density spectrum has been divided by this value and superimposed as a thin dashed line on the magnetic fluctuation spectra. The experimental data have been taken at $B_{0}=72 \mathrm{mT}$ in the density gradient region. 


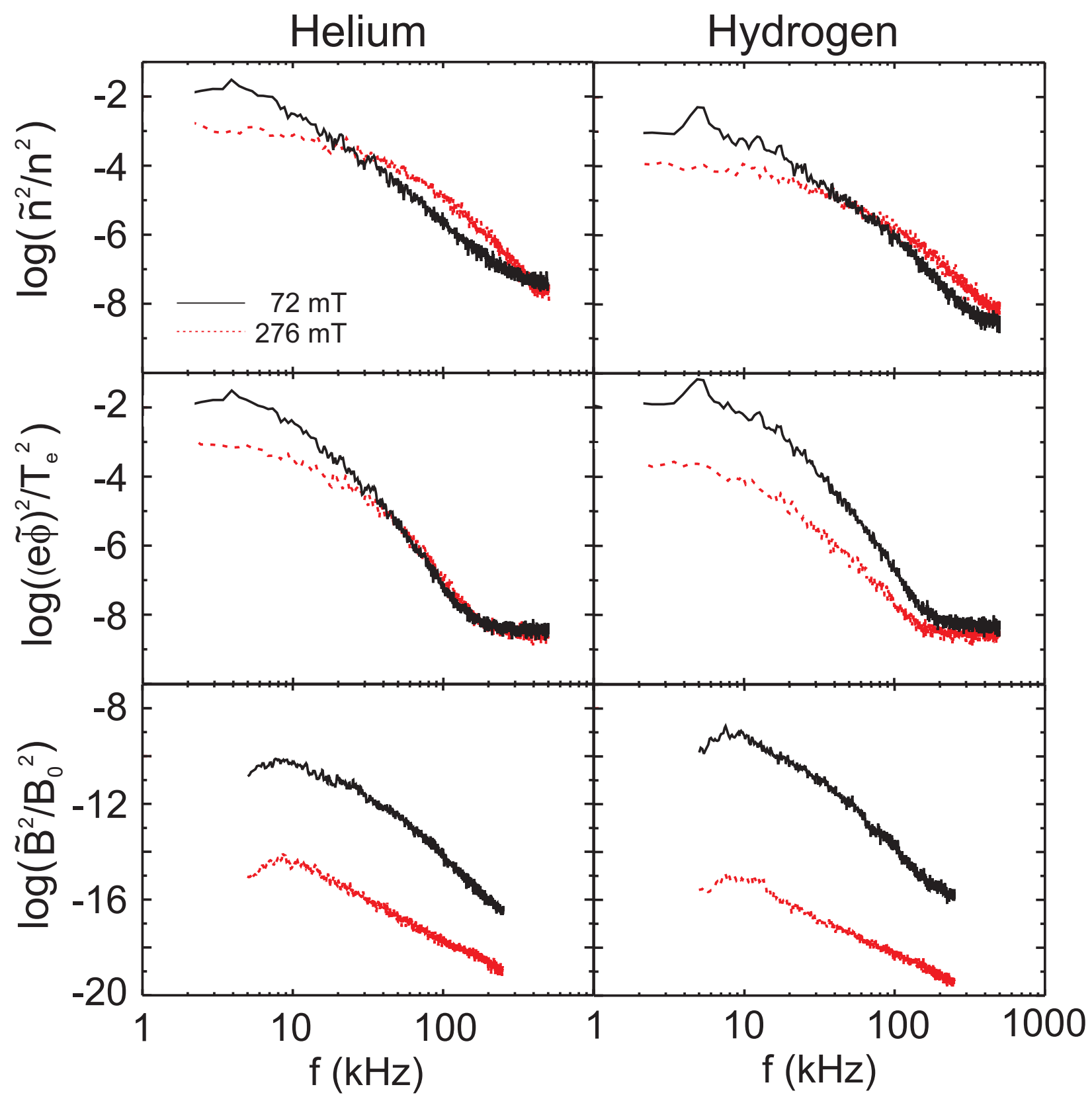

FIG. 9: Normalized density, potential and magnetic field fluctuation power spectra measured at two values of the magnetic field in helium and hydrogen. The data are shown in a way which highlights the $\beta$ scaling. The variation of both the electrostatic and magnetic spectral power on the toroidal magnetic field $B_{0}$ can clearly be seen. 